\title{
The relationship between spatial and in-store food environments and adolescent food purchasing and dietary behaviours: a systematic review
}

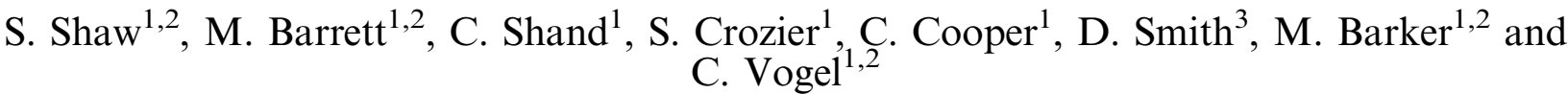 \\ ${ }^{1}$ Medical Research Council Lifecourse Epidemiology Unit, University of Southampton, Southampton, UK, \\ ${ }^{2}$ National Institute of Health Research Southampton Biomedical Research Centre, University of Southampton and \\ University Hospital Southampton NHS Foundation Trust, Southampton, UK and \\ ${ }^{3}$ School of Geography and Environmental Science, University of Southampton, Southampton, UK
}

Adolescence is a period of increased autonomy over decision making, including food choices, and increased exposure to factors outside the home. This combination may mean the physical food environment plays an important role in adolescents' independent food purchasing and dietary behaviours. This review aimed to understand how spatial access to food outlets, and environments inside food outlets, were associated with adolescent food purchasing and dietary behaviours.

Six databases were searched using MESH and free text terms. Titles and abstracts were screened by one reviewer. Studies were considered to meet the inclusion criteria if they encompassed: i) a participant sample which had a mean age between 11-18 years, ii) were conducted in real-life settings in high income countries, iii) included an exposure or intervention which related to spatial and in-store food environments, and iv) had an outcome relating to food purchasing or dietary intake. If eligible, two reviewers performed data extraction and assessed each article for risk of bias in relation to the research questions using predefined criteria based on guidelines from the Centre for Reviews and Dissemination ${ }^{(1)}$. The heterogeneous nature of these studies meant meta-analyses were not possible. In line with recommendations from Cochrane ${ }^{(2)}$, results were synthesised using a vote counting technique and effect direction plots which recorded the direction of the effect in relation to the expected relationship with health. PROSPERO registration: 42019156500.

The search yielded 4,826 potential articles; 32 observational and no intervention studies met the inclusion criteria. Two studies were classified as having a high risk of bias, 23 as moderate risk or bias and seven as low risk of bias. In the twelve studies that assessed adolescents' spatial access to food outlets classified as healthy, results did not show consistent associations with dietary outcomes. None of these studies considered adolescent food purchasing as an outcome. Twenty-eight studies assessed adolescents' spatial access to food outlets classified as unhealthy, with the majority $(n=17 / 28,61 \%)$ reporting results in the expected direction suggesting greater exposure to unhealthy food outlets was associated with poorer food purchasing and dietary behaviours. Inconsistent results were observed across the five studies investigating associations between the environment within food outlets and adolescent dietary behaviours. No studies investigated associations between the in-store environment and food purchasing.

Evidence suggests increased exposure to unhealthy food outlets is associated with increased purchasing and consumption of less healthy food in adolescents. There is limited research describing associations between exposure to healthy food outlets, the in-store environment, and adolescent food-related behaviours, particularly adolescent food purchasing. Further research in these areas may help to identify possible interventions and policy strategies to improve the diet in adolescent populations.

\section{References}

1. Centre for Reviews and Dissemination. Systematic Reviews: CRD's guidance for undertaking reviews in health care. 3 ed. York: University of York; 2008.

2. McKenzie J, Brennan S. et al. (2019) eds. Cochrane Handbook for Systematic Reviews of Interventions version 6.0. Cochrane. 